\title{
A Supportive Therapeutic and Diagnostic Modality: Sleep Deprivation
}

\author{
Pinar Guzel Ozdemir ${ }^{1 *}$, Ercan Atilla ${ }^{1}$ \\ ${ }^{1}$ Yuzuncu Yil University, Medicine Faculty, Department of Psychiatry, Van, Turkey
}

\section{Dear Editor,}

Sleep deprivation (SD) is described as a reduced ability to maintain nocturnal sleep, with increased wakefulness and totally or partial deficiencies in all of the sleep stages (Pilkington, 2013). Stressful life events often cause to sleep restriction. There is also available the beneficial use of SD in medical fields. SD is not only practiced to assess the effects of sleep loss on the behavior, biological and psychological changes it is also used for a complementary method to the treatment and diagnosis for some disorders. The best known of these, $S D$ is an effective and rapid method for depressive disorders. Firstly, SD was offered by a German psychiatrist Walter Schulte origin of his experiences that a sleepless night was followed by a substantial improvement in depression on the following day (Giedke \& Schwärzle, 2002). Then Plug and Tölle performed systematic investigations and several studies followed them (Pflug \& Tolle, 1971, Leibenluft \&Wehr, 1992). It was declared that SD affects positively in any kind of depressive disorders regardless of age. The mechanism of SD can be expressed as the monoaminergic neurotransmission increasing serotonin, noradrenaline and dopamine

*Correspondence: pguzelozdemirayahoo.com

Pinar Guzel Ozdemir, Yuzuncu Yil University, Medicine Faculty, Department of Psychiatry, Van, Turkey. Tel: +90 432215 0473, Fax:+90 4322167519

\section{Sleep and Hypnosis Journal homepage: www.sleepandhypnosis.org}

neurotransmission, alterations in the immune and endocrine systems and functional connectivity in the brain areas (Selvi et al., 2015, Dopierała \& Rybakowski, 2015).

SD is practicable in several ways such as total sleep deprivation (TSD), selective REM-sleep deprivation, latepartial sleep deprivation and early-partial sleep deprivation. Approximately $60 \%$ of the patients show clinical improvement the day immediately after the TSD night but after the recovery sleep $50 \pm 80 \%$ of SDresponders relapse (Pilkington, 2013). It is still not obvious how many hours of sleep deprivation are needed accurately antidepressant effect (Dopierała \& Rybakowski, 2015). On the other hand, it is demonstrated that the antidepressant effect is connected to SD in the second half of the night (Dopierała \& Rybakowski, 2015).

Chronic partial sleep deprivation is common in everyday life depending on the requirements of industrial and economic life. Therewithal SD induces a variety of physiological and neurobehavioral changes including cognitive functions like attention, working memory, and decision making, daytime sleepiness, fatigue, drowsiness and hypomanic or manic reactions. It effects on autonomic, biochemical, hormonal, and immunologic functions (Wirz-Justice et al., 2013) In a recent study, it is investigated the effects of SD on dissociation and mood, and its relationship with biochemical changes. For this purpose total one night SD was performed on a total of thirty-two healthy volunteers. Cortisol, dehydroepiandrosterone-sulfate (DHEA-S) and thyroid function test levels were measured 
before and after SD. At the end of the study it was found that one night SD causing to enhance the dissociative symptoms, reduce in depressive mood, and to lower the tendency of suppressing the undesirable thoughts. Among the biochemical parameters, thyroid stimulating hormone (TSH), fT3, fT4, and DHEA-S levels were increasing after the SD (Selvi et al., 2015).

A significant impact of SD is provoking epileptic seizures in individuals predisposed. Therefore SD has been used for years in electroencephalography (EEG) as a practice for activating epileptiform discharges in patients with a robust doubt of epilepsy (Renzel et al., 2016). SD- EEG is widely used in neurology practice and it increases the sensitivity of a routine EEG. A SD-EEG involves staying awake for a full night, and then EEG will be recorded for around one hour while the patient is drowsy and/or falling asleep. It was observed that

\section{References}

Dopierała, E., \& Rybakowski, J. (2015). Sleep deprivation as a method of chronotherapy in the treatment of depression. Psychiatria Polska, 49(3), 423-433.

Giedke, H., \& Schwärzler, F. (2002). Therapeutic use of sleep deprivation in depression. Sleep Medicine Reviews, 6 (5), 361377.

Giorgi, F.S., Guida, M., Caciagli, L., Maestri, M., Carnicelli, L., Bonanni, E., \& Bonuccelli, U. (2014). What is the role for EEG after sleep deprivation in the diagnosis of epilepsy? Issues, controversies, and future directions. Neuroscience \& Biobehavioral Reviews, 47, 533-548.

Leibenluft, E., \& Wehr T. (1992). Is sleep deprivation useful in the treatment of depression? American Journal of Psychiatry, 149(2), 159-168

Pflug, B. \& Tolle, R. (1971). Disturbance of the 24-hour rhythm in endogenous depression and the treatment of endogenous depression by sleep deprivation. International Pharmacopsychiatry, 6, 187. relatively short EEGs after partial SD seem to provide a diagnostic yield which is similar to that obtained after 24 $h$ of SD and a longer EEG. SD-EEG protocols is also necessary to fully explore the role of SD- EEG itself because of many unresolved issues about the pathophysiology of the effects of SD on EEG characteristics and seizure recurrence in epilepsy (Giorgi et al., 2014).

As a result SD may be practicable for many different areas in clinical applications. It is used as a chronotherapeutic for depression, can be utilized to reveal the anticipated endophenotypes and confirm the suspected diagnosis. According to our view, although SD is widely used, the trials on the SD are not enough or obsolete. It is required therapeutic and diagnostic SD researches to evaluate the short and long-term effects of this method.

Pilkington, S. (2013). Causes and consequences of sleep deprivation in hospitalised patients. Nursing Standard, 27(49), 35-42.

Renzel, R., Baumann, C.R., \& Poryazova, R. (2016). EEG after sleep deprivation is a sensitive tool in the first diagnosis of idiopathic generalized but not focal epilepsy. Clinical Neurophysiology, 127(1), 209-213.

Selvi, Y., Kılıç, S., Aydın, A., \& Özdemir, P.G. (2015). The effects of sleep deprivation on dissociation and profiles of mood, and its association with biochemical changes. Archives of Neuropsychiatry, 52(1), 83-88.

Wirz-Justice, A., Benedetti, F., \& Terman, M. (2013). Chronotherapeutics for affective disorders. A clinician's manual for light and wake therapy. Basel: Karger. 